\title{
THE AUTOMORPHISM TOWER PROBLEM
}

\author{
SIMON THOMAS ${ }^{1}$
}

\begin{abstract}
It is shown that the automorphism tower of an infinite centerless group terminates. For each ordinal $\alpha$, a group is constructed whose automorphism tower terminates in exactly $\alpha$ steps.
\end{abstract}

Let $G$ be a centerless group. Then the automorphism tower of $G$ is defined inductively by

$$
\begin{gathered}
G_{0}=G, \\
G_{\alpha} \hookrightarrow \text { Aut } G_{\alpha}=G_{\alpha+1}, \quad \text { via the natural map, } \\
G_{\lambda}=\bigcup_{\alpha<\lambda} G_{\alpha} \quad \text { when } \lambda \text { is a limit ordinal. }
\end{gathered}
$$

The automorphism tower is said to terminate if there is an ordinal $\alpha$ such that $G_{\alpha}=G_{\beta}$ for all $\beta>\alpha$. A classical result of Wielandt $[\mathbf{7}]$ says that if $G$ is finite then the automorphism tower terminates after finitely many steps. Rae and Roseblade [3] showed that this is also true if $G$ is a Cernikov group. If $G$ is a polycyclic group, then Hulse [2] has proved that the automorphism tower terminates in at most countably many steps. Whether the automorphism tower of an arbitrary centerless group terminates has been an open question for some time. This is resolved by the following theorem:

THEOREM 1. If $G$ is an infinite centerless group, then the automorphism tower of $G$ terminates in at most $\left(2^{|G|}\right)^{+}$steps.

Throughout this paper cardinals are taken to be initial ordinals. If $\kappa$ is a cardinal, then $\kappa^{+}$denotes the next greatest cardinal. As usual, $|X|$ is the cardinality of the set $X$.

The proof of Theorem 1 makes use of the fact that each successor cardinal $\kappa^{+}$is a regular cardinal. This means that if $X$ is a set of cardinality $\kappa^{+}$then $X$ cannot be expressed as a union of $\theta<\kappa^{+}$sets, $X=\bigcup_{\alpha<\theta} X_{\alpha}$, with $\left|X_{\alpha}\right|<\kappa^{+}$for all $\alpha<\theta$.

Proof of Theorem 1. By Lemma 8.1.1 of Hulse [2], $C_{G_{\alpha}}(G)=1$ for all ordinals $\alpha$. If $\varphi \in N_{G_{\alpha}}(G)$, then $\varphi \mid G=\varphi_{1} \in G_{1}$, so $\varphi \varphi_{1}^{-1} \in C_{G_{\alpha}}(G)=1$ and $\varphi=\varphi_{1}$. It follows that $N_{G_{\alpha}}(G)=G_{1}$ for all $\alpha \geq 1$. Thus, $\left[G_{\alpha+1}: G_{1}\right]$ equals the cardinality of the set of all conjugates of $G$ in $G_{\alpha+1}$. Each of the latter is contained in $G_{\alpha}$; thus,

$$
\left[G_{\alpha+1}: G_{1}\right] \leq\left|G_{\alpha}\right|^{|G|}
$$

and

$$
\left|G_{\alpha+1}\right| \leq\left|G_{1}\right| \cdot\left|G_{\alpha}\right|^{|G|}=\left|G_{\alpha}\right|^{|G|}
$$

Received by the editors May 15, 1984.

1980 Mathematics Subject Classification. Primary 20E36.

${ }^{1}$ Alexander von Humboldt Research Fellow, 1983/84. 
An easy induction shows that

$$
\left|G_{\alpha}\right| \leq 2^{|G|}
$$

for all $\alpha<\lambda=\left(2^{|G|}\right)^{+}$.

Now, assume that the automorphism tower does not terminate in less than $\lambda$ steps. Then $\left|G_{\lambda}\right|=\lambda$.

Let $\varphi \in$ Aut $G_{\lambda}$. Using the fact that $\lambda$ is a regular cardinal, it is easily shown that there is an $\alpha<\lambda$ such that $G_{\alpha}^{\varphi}=G_{\alpha}$; indeed, the set $C$ of such $\alpha$ is unbounded.

For each $\alpha \in C$ we have $\varphi \mid G_{\alpha} \in$ Aut $G_{\alpha}=G_{\alpha+1}$ so that $\varphi\left\lceil G_{\alpha}=i_{g_{\alpha}} \mid G_{\alpha}\right.$, where $i_{g_{\alpha}}$ is the inner automorphism of $G_{\lambda}$ induced by some $g_{\alpha} \in G_{\alpha+1}$. If $\alpha, \beta \in C$ then $g_{\alpha} g_{\beta}^{-1} \in C_{G_{\lambda}}(G)$ and $g_{\alpha}=g_{\beta}$. Since $C$ is unbounded, it follows that $\varphi$ is an inner automorphism of $G_{\lambda}$. Therefore, the automorphism tower of $G$ terminates at $G_{\lambda}$.

COROLLARY. An arbitrary infinite group $G$ can be embedded as an ascendant subgroup of a complete group $G^{*}$ such that $\left|G^{*}\right| \leq\left(2^{|G|}\right)^{+}$.

ProOF. By a standard procedure, embed $G$ subnormally in a centerless group $G_{1}$ such that $|G|=\left|G_{1}\right|$. Now, embed $G_{1}$ in the terminal group of its automorphism tower.

It is not known yet whether $\left(2^{|G|}\right)^{+}$is the best possible bound. The next result shows that the bound does depend on the cardinality of $G$.

THEOREM 2. Suppose that $\alpha$ is an ordinal and $\kappa=\max \{\omega,|\alpha|\}$. Then there exists a centerless group $G$ of cardinality $\kappa$ such that the automorphism tower of $G$ terminates in exactly $\alpha$ steps.

First, another definition is required. If $H$ is a subgroup of $G$, then the normalizer tower of $H$ in $G$ is defined inductively by

$$
\begin{gathered}
N_{0}(H)=H \\
N_{\alpha+1}(H)=N_{G}\left(N_{\alpha}(G)\right) \\
N_{\lambda}(H)=\bigcup_{\alpha<\lambda} N_{\alpha}(H), \quad \text { when } \lambda \text { is a limit ordinal. }
\end{gathered}
$$

LEMMA. Let $\alpha$ be an ordinal. There exist groups $H(\alpha)<F(\alpha)$ such that

(a) $|F(\alpha)| \leq \max \{\omega,|\alpha|\}$,

(b) the normalizer tower of $H(\alpha)$ in $F(\alpha)$ terminates in exactly $\alpha$ steps; further, $N_{\alpha}(H(\alpha))=F(\alpha)$.

Proof. The groups $H(\alpha)$ and $F(\alpha)$ are constructed inductively . Define

$$
H(1)=\operatorname{Alt}(4)<\operatorname{Sym}(4)=F(1) \text {. }
$$

If $\alpha=\beta+1$, let $F(\alpha)=F(\beta)$ wr $C_{2}$ and take $H(\alpha)$ to be the subgroup $H(\beta) \times 1$ of the base group. If $\alpha$ is a limit ordinal, define

$$
H(\alpha)=\operatorname{Dr}_{\beta<\alpha} H(\beta)<\operatorname{Dr}_{\beta<\alpha} F(\beta)=F(\alpha)
$$

(Dr denotes the restricted direct product).

PROOF OF THEOREM 2. Fried and Kollár [1] have shown that there exists a field $K$ of cardinality $\kappa$ such that Aut $K=F(\alpha)$. By Schreier and van der Waerden [6],

$$
\text { Aut } \operatorname{PSL}(2, K)=\operatorname{P\Gamma L}(2, K)=\operatorname{PGL}(2, K) \rtimes F(\alpha) \text {. }
$$


We claim that if $G=\operatorname{PGL}(2, K) \rtimes H(\alpha)$, then for each $\beta \leq \alpha$,

$$
G_{\beta}=\operatorname{PGL}(2, K) \rtimes N_{\beta}(H(\alpha)),
$$

the $\beta$ th term of the normalizer tower of $G$ in Aut PSL $(2, K)$. Suppose that this is true for $\beta<\alpha$ and consider $G_{\beta+1}=\operatorname{Aut} G_{\beta}$. Since $\operatorname{PSL}(2, K)$ is the unique minimal normal subgroup of $G_{\beta}$, there is an induced homomorphism

$$
\varphi: \operatorname{Aut} G_{\beta} \rightarrow \operatorname{AutPSL}(2, K),
$$

which is easily seen to be an embedding (e.g., Robinson [4, 13.5.2]). Note that $\varphi \uparrow G_{\beta}$ is the identity map. So, identifying Aut $G_{\beta}$ with its image under $\varphi$, it follows that Aut $G_{\beta}=\operatorname{PGL}(2, K) \rtimes N_{\beta+1}(H(\alpha))$. Clearly no problems arise for limit ordinals. Thus, the automorphism tower of $G$ terminates in exactly $\alpha$ steps.

Schenkman [5] proved that if $L$ is a finite-dimensional centerless Lie algebra, then the tower of derivation algebras of $L$ terminates after finitely many steps. A routine modification of the above argument shows that the tower of derivation algebras of an infinite-dimensional centerless Lie algebra $L$ terminates in at most $\left(2^{\operatorname{dim} L}\right)^{+}$steps.

\section{REFERENCES}

1. E. Fried and J. Kollár Automorphism groups of fields, Universal Algebra (E. T. Schmidt et al., eds.), Colloq. Math. Soc. Janos Boyali, vol. 24, 1981, pp. 293-304.

2. J. A. Hulse, Automorphism towers of polycyclic groups, J. Algebra 16 (1970), 347-398.

3. A. Rae and J. E. Roseblade, Automorphism towers of extremal groups, Math. Z. 117 (1970), 70-75.

4. D. J. S. Robinson, A course in the theory of groups, Springer-Verlag, New York, 1982.

5. E. V. Schenkman, $A$ theory of subinvariant Lie algebras, Amer. J. Math. 73 (1951), 453-474.

6. O. Schreier and B. L. van der Waerden, Die Automorphismen der projektiven Gruppen, Abh. Math. Sem. Univ. Hamburg 6 (1928), 303-322.

7. H. Wielandt, Eine Verallgemeinerung der invarianten Untergruppen, Math. Z. 45 (1939), 209-244.

Mathematisches Institut, Albert-Ludwigs-Universitat, Freiburg I. BR

Current address: Department of Mathematics, Yale University, New Haven, Connecticut 06520 